\title{
TURBULENT MIXING IN MULTI-NOZZLE INJECTION TUBULAR MIXER*
}

\author{
Osato MIYAWAKI, Hiroo TSUJIKAWA And Yuzo URAGUGHI \\ Department of Chemical Engineering, University of Tokyo, Tokyo
}

\begin{abstract}
Turbulent mixing in this type mixer is experimentally investigated. Probability density distribution function of $c^{\prime}$ is measured by using digital counter and proves to be normally distributed. This fact affirms the basis of Toor's theory. Concentration spectrum has a narrow $-5 / 3$ power region. Toor's theory concerning the relation between concentration fluctuation and conversion of very rapid reaction is experimentally proved. Initial mixing rate is studied on the basis of it. Dependence of the mixing time constant on $\bar{U}, \bar{\alpha}, d_{N}$ and $D_{m}$ are measured. Their effects are generally explained by modified Corrsin's equation.
\end{abstract}

\section{Introduction}

Turbulent mixing between two miscible fluids is one of the most universal phenomena in chemical engineering. However, there still remain many problems to be solved regarding it. A multi-nozzle injection mixer involves turbulent mixing in its simplest form. Consequently, it is useful in understanding the basic aspects there of to study such a mixer.

Gibson and Schwarz ${ }^{4)}$ investigated grid turbulence in a duct, where they made measurements in connection with the velocity and the scalar field, confirmed the existence of Kolmogoroff's universal equilibrium range and the inertialsubrange in the velocity field and experimentally proved Batchelor's -1 power law in the scalar field.

Keeler et al. ${ }^{\text {) }}$ and Vassilatos and Toor ${ }^{13)}$ investigated multi-nozzle injection tubular mixers and experimentally proved Toor's theory ${ }^{12)}$, which relates the intensity of the concentration fluctuation of nonreacting mixing with the conversion of very rapid reaction.

Miyairi et al. ${ }^{9)}$ studied promotion and control of mixing in a similar type mixer by using various mesh grids.

Also in this report, the multi-nozzle injection tubular mixer is taken up. The turbulent concentration field is investigated and some measurements are performed. The initial mixing rate is measured and analysed quantitatively by Corrsin's theory ${ }^{2,3)}$.

\section{Theoretical Background}

\section{1 Structure of turbulent concentration field}

Turbulent concentration field is characterized by fluctuations of concentration $c^{\prime}$, the value of which varies with place and time. So inevitably we must deal with the statistical value of $c^{\prime}$. Two types of statistics are

\footnotetext{
* Received on June 23, 1973

Presented at the 5th Autumn Meeting of The Soc. of Chem. Engrs., Japan, October, 1971, Osaka

T113 東京都文京区本郷7-3-1

東京大学工学部化学工学科 宮脇長人
}

considered hereafter.

One is statistics for wave number of $c^{\prime}$. This is known as spacial spectrum of concentration fluctuation $G(k)$ and was dealt with by many investigators ${ }^{5)}$. The relation between $\overline{c^{\prime 2}}$ and $G(k)$ is

$$
\overline{c^{\prime 2}}=\int_{0}^{\infty} G(k) \mathrm{d} k
$$

In the inertial subrange, it has the well-known form

$$
G(k)=\text { const } \times k^{-5 / 3} \quad\left(k_{e} \ll k \ll k_{K}\right)
$$

where $k_{e}$ and $k_{K}$ are, respectively, the characteristic wave number of large eddies and Kolmogoroff's wave number. In the higher wave number region, where the molecular diffusion becomes important, there is Batchelor's theory ${ }^{1)}$.

$$
\begin{aligned}
G(k)=\text { const } \times(1 / k) \times \exp \left(-2 k^{2} / k_{B}^{2}\right) \\
\left(S c \gg 1, k_{K} \ll k\right)
\end{aligned}
$$

Other statistics for $c^{\prime}$ have been less dealt with than those above. The probability density distribution function $P\left(c^{\prime}\right)$ of concentration fluctuation can be considered in a turbulent field.

$$
\begin{aligned}
& \int_{-\infty}^{+\infty} P\left(c^{\prime}\right) \mathrm{d} c^{\prime}=1 \\
& \int_{-\infty}^{+\infty} c^{\prime} P\left(c^{\prime}\right) \mathrm{d} c^{\prime}=0 \\
& \int_{-\infty}^{+\infty} c^{\prime 2} P\left(c^{\prime}\right) \mathrm{d} c^{\prime}=c^{\prime 2}
\end{aligned}
$$

It was introduced by Hawthorne et $\left.a l .^{6}\right)$, after which Toor ${ }^{11)}$ composed his theory using this function. Tsujikawa and Uraguchi ${ }^{12}$ pointed out the importance of the $P\left(c^{\prime}\right)$ when the concentration of a turbulent field is measured by the electric conductivity method. Thus information on $P\left(c^{\prime}\right)$ is of practical use, although there seem to be few measurements of it.

\section{2 Toor's theory}

Toor ${ }^{11)}$ proposed a relation between $c^{\prime 2}$ in nonreacting mixing and the conversion of very rapid reaction $F_{R}$, using the assumption that $P\left(c^{\prime}\right)$ is a normal distri- 
bution.

$$
P\left(c^{\prime}\right)=\frac{1}{\sqrt{2 \pi} \sigma} \exp \left(-\frac{c^{\prime 2}}{2 \sigma^{2}}\right)
$$

According to his theory, if two reactants are supplied equivalently, the relation between them is

$$
1-F_{R}=\text { const } \times \sqrt{\overline{c^{\prime 2}}}
$$

\section{3 Mixing rate}

The scalar decay equation in isotropic turbulent field is

$$
-\left(\mathrm{d} \overline{c^{2}} / \mathrm{d} t\right)=12 D_{m} \times\left(\overline{c^{2}} / l_{c}^{2}\right)
$$

This is regarded as the mixing rate equation. Corrsin ${ }^{2}$, 3) assumed a steady turbulence, where the miçroscale in the velocity field is constant and so is the microscale in the concentration field, although $\overline{c^{\prime 2}}$ is decaying. Then, Eq.(9) can be easily integrated.

$$
\begin{aligned}
& \left.\overline{c^{\prime 2}}(t)=\overline{\left(c^{\prime 2}\right.}\right)_{i} \exp \left(-t / t_{M}\right) \\
& t_{M}=l_{c}^{2} / 12 D_{m}
\end{aligned}
$$

The concept of steady turbulence may fundamentally be inconsistent with the isotropy. But with high Reynolds number, Corrsin assumed its validity as an approximation. He further calculated the mixing time constant $t_{M}$ assuming Eqs.(2) and (3) as the concentration spectrum.

$$
t_{M} \fallingdotseq \frac{1}{2}\left(\frac{3}{\varepsilon^{1 / 3} k_{0 c}^{2 / 3}}+(\nu / \varepsilon)^{1 / 2} \log (S c)\right)
$$

\section{Experimental Equipment}

The flow system is schematically shown in Fig. 1(a). The constant head in the overflow tank supplies a steady flow. The mixer is a $40-\mathrm{mm}$ I.D. Lucite pipe which has 24 nozzles at the inlet. Half of these nozzles were assigned to one flow and the other half to the other. These were located alternately, as shown in Fig. 1 (b). The nozzle diameters used were 2.5, 3.2, 4.0 and $5.0 \mathrm{~mm}$. For the case of nonreacting mixing, the two flows to be mixed contain respectively two kinds of $\mathrm{NaNO}_{3}$ solutions with different concentration. It is usual in such an experiment that one of the flows contains pure water only. But this might cause an experimental error because of fluctuations smaller than the probe scale ${ }^{12)}$. In the test of the dependence of the mixing rate on the diffusion coefficient, $\mathrm{HCl}$ solution was used.

The detection of concentration was performed by means of the electroconductivity method. A small platinum electrode, with one pole being $0.1 \mathrm{~mm} \phi$ wire and the other of much larger scale, was used. Therefore the resolving power of the electrode was mainly controlled by the smaller pole. The block diagram of the measuring electric circuit is shown in Fig. 2. The intensity of the fluctuation $\overline{c^{\prime 2}}$ was measured by using the circuit shown in Fig. 2(a). The frequency of the oscillator was $20 \mathrm{kHz}$. At the points A, B the modulated wave came out, consisting of the oscillator wave as a carrier and

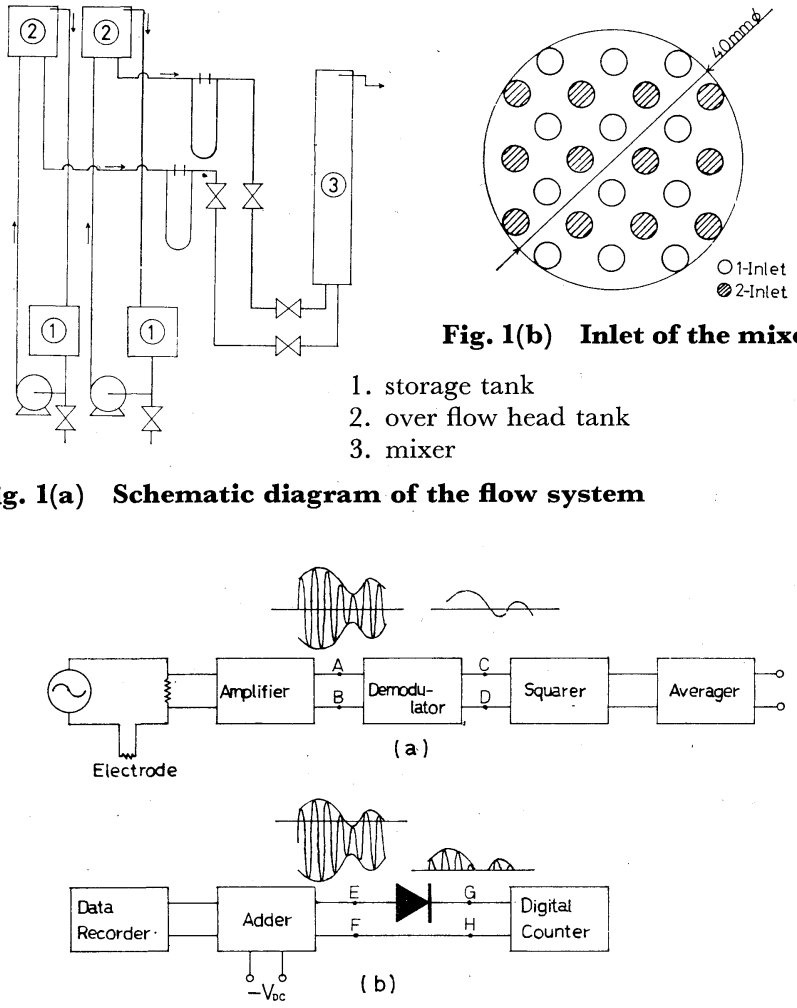

Fig. 2 Block diagram of the measuring electric circuit

the wave corresponding to the concentration fluctuation as a signal. The frequency of the former was much higher than that of the latter. This modulated wave was demodulated and the signal corresponding to $c^{\prime}$ was taken out at $\mathrm{C}, \mathrm{D}$. It wa squared and averaged. And finally $\overline{c^{\prime 2}}$ was obtained.

For the measurement of the spectrum, the signal at C, D was recorded by a tape data recorder. It was analyzed by a band-pass filter and the concentration spectrum was derived.

In the measurement of the probability density distribution function of $c^{\prime}$, the modulated wave at A, B was directly recorded by the tape recorder. The recorded data were dealt with by the circuit as shown in Fig. 2(b). In this circuit, the output at E, F was the modulated wave subtracted by the constant voltage $V_{D C}$. At $\mathrm{G}, \mathrm{H}$, its positive part only came out as pulses, which were counted by a digital counter. The value of $V_{D C}$ was systematically changed and a series of countings was performed. $P\left(c^{\prime}\right)$ was obtained from it.

In the case of very rapid reaction the neutralization reaction of $\mathrm{HNO}_{3}$ and $\mathrm{NaOH}$ was adopted for the tracer reaction. The conversion was measured by a small thermocouple which detected the temperature rise caused by the heat of reaction.

\section{Results and Discussion}

\section{1 Probability density distribution function}

In Fig. 3 the measured $P\left(c^{\prime}\right)$ is shown. It contains three data, which were measured at different axial positions. In mixing of this type, the initial form of $P\left(c^{\prime}\right)$ must be the piling of two impulse functions. 


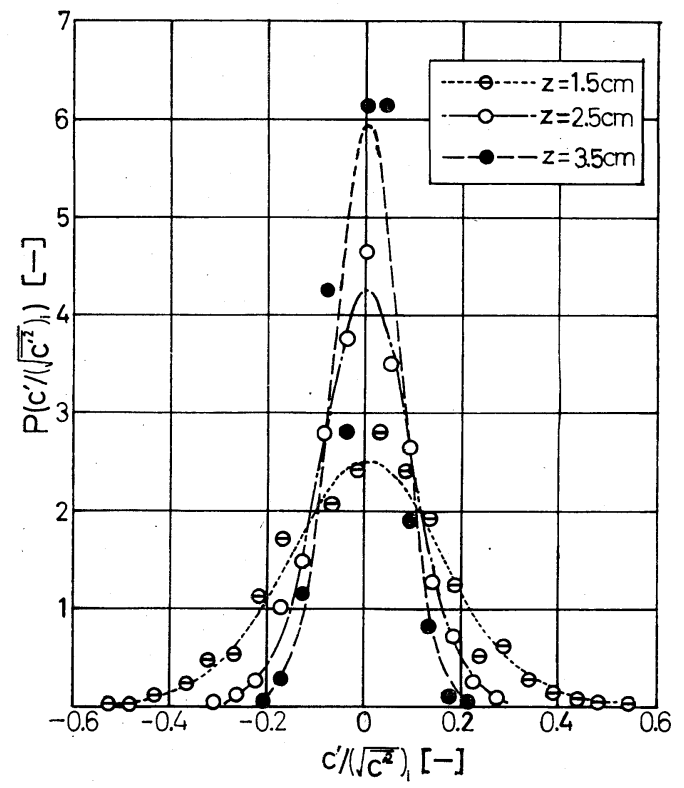

Fig. 3 Probability density distribution function of $c^{\prime}$

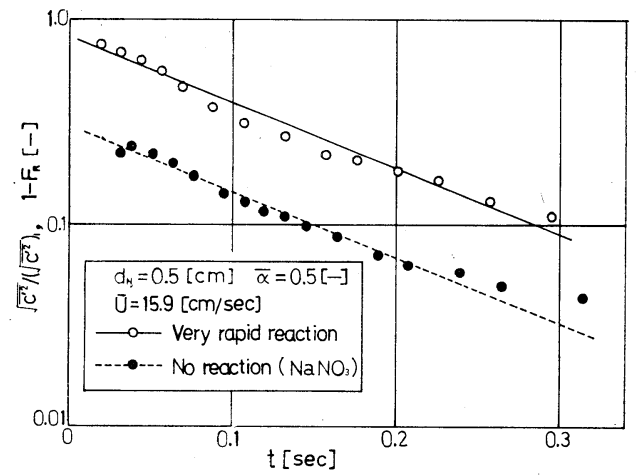

Fig. 5 Relation of the conversion of very rapid reaction and the intensity of the fluctuation with no reaction

$$
P_{i}\left(c^{\prime}\right)=\bar{\alpha} \delta\left(c^{\prime}-c_{1 i}^{\prime}\right)+(1-\bar{\alpha}) \delta\left(c^{\prime}-c_{2 i}^{\prime}\right)
$$

And it finally becomes

$$
P_{\infty}\left(c^{\prime}\right)=\delta\left(c^{\prime}\right)
$$

Between these two limiting cases, there is an intermediate state. The state shown in Fig. 3 is regarded to be in this state. The progress of the mixing is clearly expressed there. Normal distribution functions with the same variance are also shown. It seems to be in good accordance with the data. To confirm this, the skewness and the flatness were calculated for the data. The skewness was $-0.105,-0.047$ and -0.227 , respectively, corresponding to $z=1.5,2.5$ and $3.5 \mathrm{~cm}$. The flatness was 2.92, 2.99 and 2.95. In the normal distribution, the skewness is equal to 0 and the flatness is 3 . Therefore $P\left(c^{\prime}\right)$ may be sufficiently described by the normal distribution.

\section{2 Concentration spectrum}

The normalized one-dimensional spectrum is shown in Fig. 4. There seems to be a narrow region where $-5 / 3$ power law comes into existence. In the higher wave number region the resolving power of the probe may not be sufficient and so Batchelor's region couldn't be observed. The Reynolds number and the axial position were changed in the measurement. But their

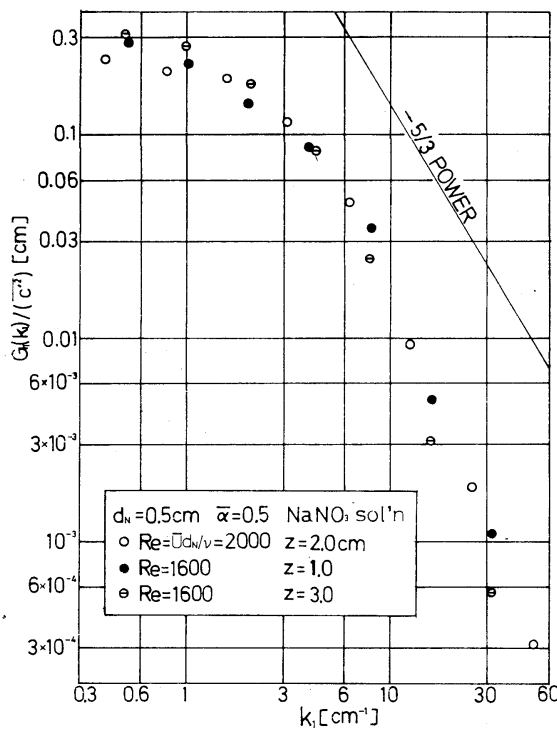

Fig. 4 One-dimensional concentration spectrum

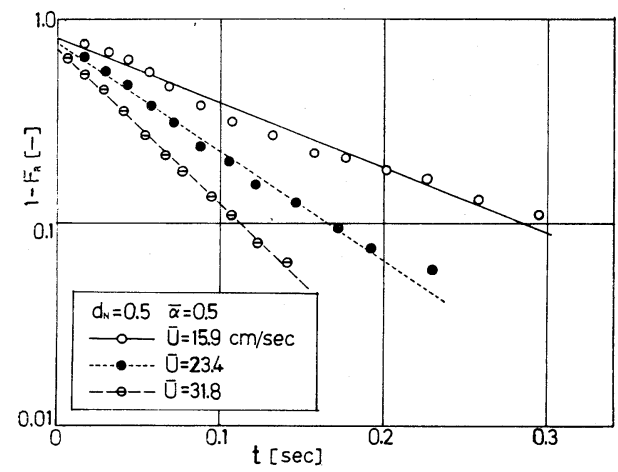

Fig. 6 Effect of the average velocity to mixing rate

effects seem to be not so large. However, the low wave number component seems to increase a little and the high wave number component to decrease a little as the mixing proceeds. The macroscale in the concentration field $L_{c}$ can be calculated by this data.

$$
L_{c}=\frac{\pi}{\overline{2 c^{\prime 2}}} \lim _{k \rightarrow 0} G_{1}\left(k_{1}\right)=\frac{\pi}{2} \times 0.3=0.5 \mathrm{~cm}
$$

\section{3 Fluctuation and very rapid reaction}

As previously mentioned there are many experimental proofs of Toor's theory. Here it was reaffirmed in Fig. 5.

\section{4 Initial character of mixing}

In Fig. 5, the linearity of the plot shows the exponential decay described by Eq.(10). This initial character of the mixing will be further investigated.

The dependence of mixing upon the average velocity $\bar{U}$ is shown in Fig. 6. The abscissa is the time $t$. If this is the axial distance $z$, the three plots in the figure will nearly superimpose one upon another. But also in that case the data for larger $\bar{U}$ decay a little faster than for smaller $\bar{U}$.

In the next place, the effect of the flow rateratio $\bar{\alpha}$ is shown in Fig. 7. These data seem to show that the larger the difference in flow rate between the two, the larger the shear. And so the intensity of the turbulence becomes large and makes mixing faster. 


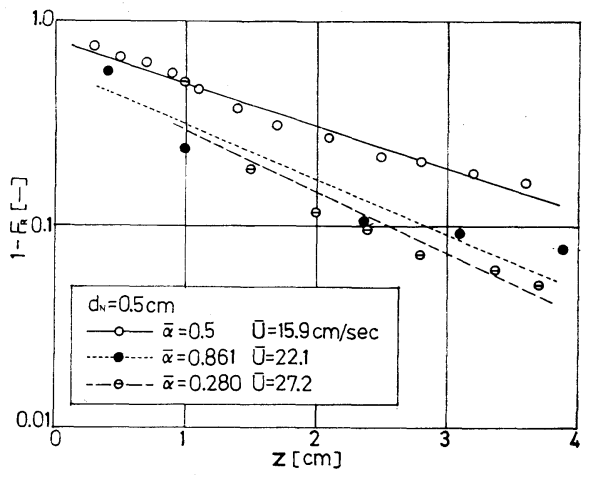

Fig. 7 Effect of the flow rate ratio to mixing rate

The dependence on nozzle diameter $d_{N}$ is shown in Fig. 8. It was expected that with the number of nozzles unchanged the mixing rate becomes larger in the finer nozzle mixer. This is proved in the figure.

In Fig. 9, the effect of the diffusion coefficient $D_{m}$ is shown. Hitherto in this section, the data of mixing were obtained from $1-F_{R}$. But in this case $\overline{c^{\prime 2}}$ was measured by using $\mathrm{HCl}$ and $\mathrm{NaNO}_{3}$ solutions. The diffusion coefficient of $\mathrm{HCl}$ and $\mathrm{NaNO}_{3}$ are $3.1 \times 10^{-5}$ and $1.5 \times 10^{-5}\left[\mathrm{~cm}^{2} / \mathrm{sec}\right]$, respectively. It is approximately a two-fold change. The mixing is faster in the case of $\mathrm{HCl}$ but there seems to be little difference in the mixing time constant $t_{M}$.

The dependence of mixing on the parameters $\bar{U}, \bar{\alpha}$, $d_{N}$ and $D_{m}$ will be discussed generally from the standpoint of the mixing-time constant. Then Corrsin's Eq. (12) attracts attention. In this equation $k_{0 c}$ can be estimated from the value of $L_{c}$ in Eq.(15).

$$
k_{0 c}=2 \pi / L_{c} \fallingdotseq 12.5 \mathrm{~cm}
$$

The energy dissipation $\varepsilon$ is an internal parameter of turbulence and was not measured in the experiment. It will be estimated from the external parameters. In the isotropic theory it is well known that

$$
-\frac{1}{u^{\prime 2}} \frac{\mathrm{d} u^{\prime 2}}{\mathrm{~d} t} \fallingdotseq k_{e} \sqrt{\overline{u^{\prime 2}}}
$$

where $k_{e}$ is the characteristic wave number of largescale eddies. It can be rewritten in the form

$$
\varepsilon \doteqdot\left(\overline{u^{\prime 2}}\right)^{3 / 2} k_{e}
$$

The value of $\overline{u^{\prime 2}}$ and $k_{e}$ may change with time or in this case with position. But these are represented by one set of characteristic values in the total mixing section. They are determined by the inlet condition because the mixing in this mixer mainly submits to this condition. The intensity $\overline{u^{\prime 2}}$ in this meaning is estimated as follows.

Here it is assumed that

$$
\left.\overline{u^{\prime 2}}=\text { const } \times \overline{\left(u^{\prime 2}\right.}\right)_{-0}
$$

where $\left(\overline{u^{\prime 2}}\right)_{-0}$ is the cross-sectional-averaged intensity of turbulence before inlet of the mixer. This is calculated from the actual velocity in each nozzle. In Fig. 1 (b) the velocity in the unshadowed nozzles is represented by $u_{1}$, and that in the shadowed one is represented by $u_{2}$. They are connected with each other

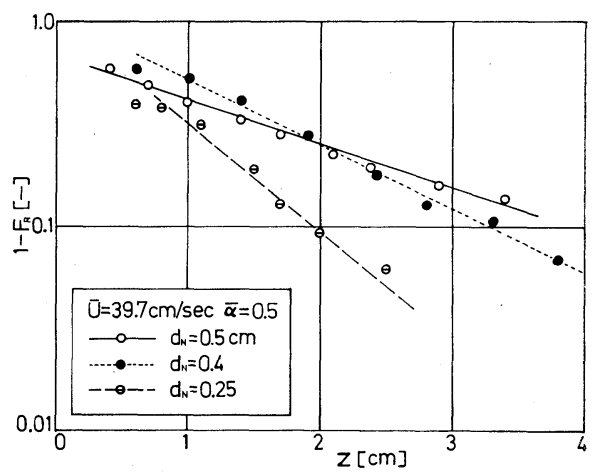

Fig. 8 Effect of the nozzle diameter to mixing rate

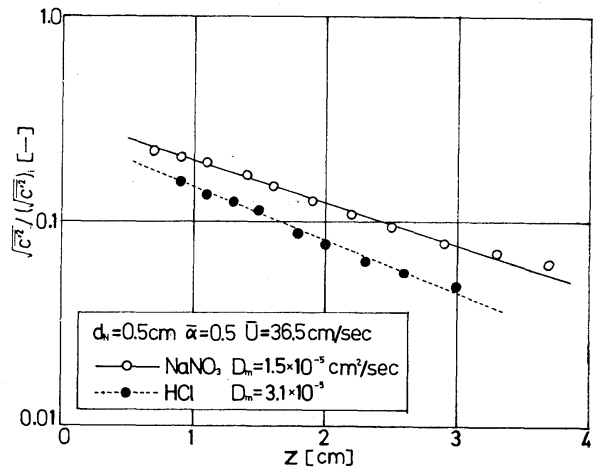

Fig. 9 Effect of the diffusion coefficient to mixing rate

through the following equations.

$$
\begin{aligned}
& u_{1} / u_{2}=\bar{\alpha} /(1-\bar{\alpha}) \\
& u_{1} f+u_{2} f=\bar{U}
\end{aligned}
$$

where $f$ is the area ratio of the section occupied by each nozzle to total sectional area in Fig. 1(b). Then area ratio of the section unoccupied by the nozzles is $1-2 f$, where the value of the velocity is zero. From these $\left(\overline{u^{\prime 2}}\right)_{-0}$ is calculated as follows.

$$
\begin{aligned}
\left(\overline{u^{\prime 2}}\right)_{-0} & =\left(u_{1}-\bar{U}\right)^{2} f+\left(u_{2}-\bar{U}\right)^{2} f+(0-\bar{U})^{2}(1-2 f) \\
& =\bar{U}^{2}\left(\left(2 \bar{\alpha}^{2}-2 \bar{\alpha}+1\right) / f-1\right)
\end{aligned}
$$

From the right side of this equation it is known that if $\bar{U}$ and $f$ are constant then $\left(\overline{u^{\prime 2}}\right)_{-0}$ takes its minimum value at $\bar{\alpha}=0.5$, and the larger the discrepancy of $\bar{\alpha}$ from 0.5 the larger $\left(\overline{u^{\prime 2}}\right)_{-0}$. It may be possible to explain the effect of $\bar{\alpha}$ shown in Fig. 8. The scale of large eddy may be determined by the nozzle diameter, and so $k_{e}$ is assumed to be

$$
k_{e}=2 \pi / d_{N}
$$

At this point the only parameter remaining is the constant in Eq.(19). To obtain it one arbitrary datum of $t_{M}$ was selected and the constant in Eq.(19) was adjusted so that $t_{M}$ calculated was consistent with that datum. The run denoted by key 1 in Fig. 10 was chosen for this purpose and it was found that

$$
\sqrt{\overline{u^{\prime 2}}}=0.133 \times \sqrt{\overline{\left(u^{\prime 2}\right)_{-0}}}
$$

With Eqs. (12), (18), (22) and (24), $t_{M}$ for all the other runs can be calculated. The large difference of these equations from Eq.(12) may be because the former include the external parameters $\bar{U}, \bar{\alpha}, f$ and $d_{N}$ instead 


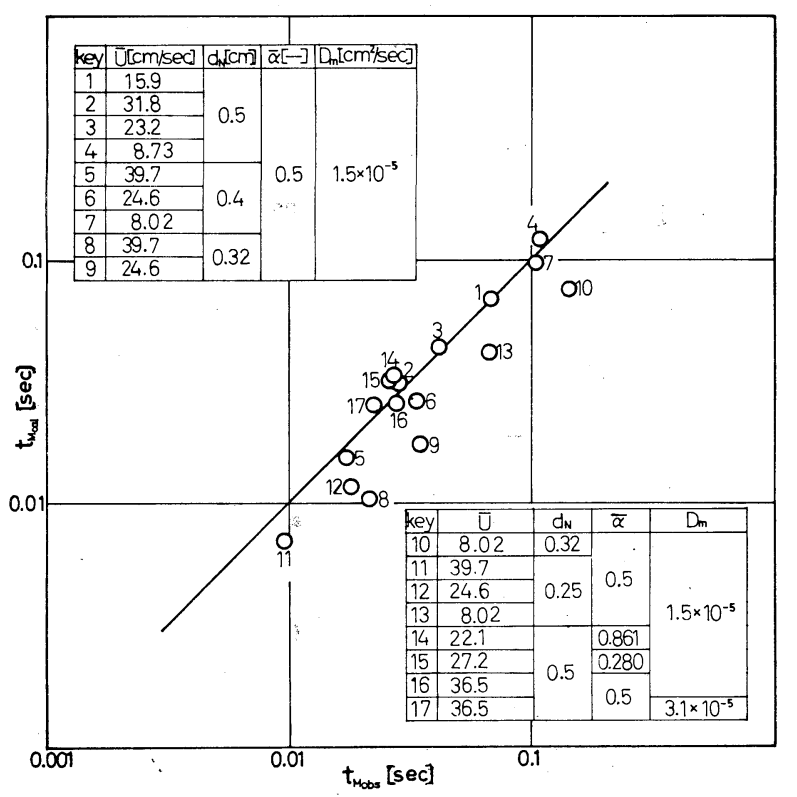

Fig. 10 Correlation between $t_{M}$ calculated and observed

of the internal parameter $\varepsilon$ in the latter. The calculated values of $t_{M}$ are plotted against the observed value in Fig. 10. The correlation seems to be fairly good.

\section{Conclusions}

Turbulent mixing in the multi-nozzleinjection tubular mixer was studied. The equations of Toor and Corrsin and the basis for their derivation were investigated. Main results obtained by the present work are as follows.

1. The probability density distribution function of $c^{\prime}$ was measured. It was proved to be the normal distribution.

2. The concentration spectrum was measured. There was a narrow region where $-5 / 3$ power law came into existence.

3. Toor's theory concerning the relation between $\overline{c^{\prime 2}}$ and $1-F_{R}$ was experimentally proved. Mixing was investigated on the basis of it. The initial decay measured in the form of $1-F_{R}$ or $\sqrt{c^{\prime 2}}$ was described by the exponential law.

4. The mixing rate was investigated in the form of $t_{M}$ with four parameters changed. They are $\bar{U}, \bar{\alpha}, d_{N}$ and $D_{m}$, and their effects were generally correlated by a modified Corrsin's equation, where the internal parameter $\varepsilon$ was replaced by the external parameters $\bar{U}, \bar{\alpha}, f$ and $d_{N}$.

\section{Nomenclature}

$=$ fluctuation of concentration from its mean value

$c_{1 i}^{\prime}, c_{2 i}^{\prime}$
$D_{m}$
$d_{N}$
$F_{R}$
$f$

initial fluctuation of concentration

corresponding to the two fluids, respectively

$=$ molecular diffusion coefficient

$=$ nozzle diameter

$=$ conversion of very rapid reaction

$=$ sectional area ratio occupied by one flow at the inlet

$G(k), G_{1}\left(k_{1}\right) \quad=$ three and one dimensional concentration spectrum, respectively

$k, k_{1}=$ wave number in three and one dimensional

$k_{B} \quad=\quad$ Batchelor's wave number $=\left(\varepsilon / \nu D_{m}^{2}\right)^{1 / 4}$

$\left[\mathrm{mol} / \mathrm{cm}^{3}\right]$

$k_{e} \quad=$ characteristic wave number of large scale eddies

$\begin{array}{ll}k_{K} & =\text { Kolmogoroff's wave number }=\left(\varepsilon / \nu^{3}\right)^{1 / 4} \\ k_{P} & =\text { characteristic wave number of probe scale }\end{array}$

$k_{0 c}=$ characteristic wave number of large scale concentration eddies

$=$ macro scale of concentration field

$\begin{array}{ll}L_{c} & =\text { macro scale of concentration field } \\ l_{c} & =\text { micro scale of concentration field }\end{array}$

$l_{P} \quad=$ probe scale

$P\left(c^{\prime}\right) \quad=$ probability density distribution function of $c^{\prime}$

$P_{i}\left(c^{\prime}\right), P_{\infty}\left(c^{\prime}\right)=P\left(c^{\prime}\right)$ at initial and final time of mixing, respectively

Re $\quad=$ Reynolds number $=\left(\bar{U} d_{N} / \nu\right)$

Sc $\quad=$ Schmidt number $=\left(\nu / D_{m}\right)$

$t \quad=z / U=$ time

$t_{M} \quad=$ time constant of mixing

$\bar{U} \quad=$ mean velocity

$u^{\prime} \quad=$ fluctuation of velocity from its mean value

$u_{1}, u_{2} \quad=$ velocity in each nozzles

$z \quad=$ axial distance $\quad[\mathrm{cm}]$

$\alpha \quad=$ flow rate ratio $\quad[-]$

$\delta \quad=$ delta function

$\varepsilon \quad=$ turbulent energy dissipation in unit mass and time

$=$ kinetic viscosity

$=$ variance of $c^{\prime}$

$\left[\mathrm{cm}^{2} / \mathrm{sec}\right]$

$\left[\mathrm{cm}^{2} / \mathrm{sec}\right]$

\section{Literature Gited}

1) Batchelor, G. K.: J. Fluid Mech., 5, 113 (1959)

2) Corrsin, S.: AIChE J., 3, 329 (1957)

3) Corrsin, S.: ibid., 10, 870 (1964)

4) Gibson, C. H. and W. H. Schwarz: J. Fluid Mech., 16, 365, (1963)

5) Hinze, J. O.: “Turbulence", McGraw Hill (1959)

6) Hawthorne, W. R., D. S. Weddel and H. G. Hottel:" 3rd Symp. on Combustion, Flame and Explosion Phenomena", p. 266, Williams and Wilkins (1949)

7) Keeler, R. N., E. E. Petersen and J. M. Prausnits: AIChE J., 11, 221 (1965)

8) Kolmogoroff, A.: Comptes Rendes de l'Academie des Sciences de l'URSS, XXXX, 301 (1941)

9) Miyairi, Y., M. Kamiwano and K. Yamamoto: Kagaku Kōgaku, 34, 1315 (1970)

10) Sato, Y., M. Kamiwano and K. Yamamoto: Kagaku Kögaku, 34, $104(1970)$

11) Toor, H. L.: AIChE J., 8, 70 (1962)

12) Tsujikawa, H. and Y. Uraguchi: J. Chem. Eng. Japan, 6, 92 (1973)

13) Vassilatos, G. and H. L. Toor: AIChE J., 11, 666 (1965) 